\title{
Ước tính sức chịu tải động cực hạn của móng nông trên nền đất yếu bằng phương pháp tiếp cận - phương pháp tựa tĩnh, cân bằng giới hạn và động lực học
}

\section{Lê Bảo Quốc ${ }^{1}$}

${ }^{1}$ Đại học xây dựng Miền Tây

\section{TỪ KHOÁ}

Tải trọng động

Động đẩt

Sức chịu tải động cực hạn

Móng nông

\begin{abstract}
TÓM TÁT
Tính toán thiết kế kết cấu móng công trình chịu tải trọng và ảnh hưởng sự hóa lỏng của nền đất là vấn đề đã thu hút các nhà nghiên cứu trong nhiều năm. Lý thuyết tính toán xác định sức chịu tải động cực hạn của móng phần lớn phân tích để thiết kế móng nông dưới tải trọng địa chấn dựa trên giả định rằng các vùng hư hỏng trong đất xảy ra dọc theo bề mặt hư hỏng chịu tác dụng tải trọng tĩnh, đây là tiếp cận giả tĩnh. Thực tế để tính toán sức chịu tải dưới tác động của địa chấn thông thường tăng lên một hệ số 1,25 theo khuyến nghị trong một số quy chuẩn trên thế giới. Và một vài nhà nghiên cứu đã phát triển các hệ số sức chịu tải động $(\mathrm{Nc}, \mathrm{Nq}, \mathrm{N} \gamma)$ dưới tải trọng động để thiết lập một mô hình toán học nhằm xác đ ịnh ước tính sức chịu tải cực hạn dựa trên giả định rằng lực địa chấn có bản chất là tựa tĩnh và đồng thời theo phương pháp động lực học có xét đến tăng thêm bởi khối lượng đất rung. Bài báo trình bày tiếp cận phương pháp tính toán thiết kế móng nông chịu tải trọng động theo các phương pháp tựa tĩnh, cân bằng giới hạn và động lực học.
\end{abstract}

\begin{abstract}
The calculating design of the foundation structures under the dynamic load and the liquefaction effect of the ground is a problem that has attracted researchers for many years. The calculation theory for determining the ultimate dynamic load capacity of the foundation is largely analyzed for the design of shallow foundations under seismic loads based on the assumption that the failure zones in the soil occur along the affected failure surface of static load, this is a pseudo-static approach. In fact, to calculate the bearing capacity under seismic action, it is usually increased by a factor of 1.25 as recommended in some international standards. And several researchers have developed dynamic load coefficients (Nc, Nq, N $\gamma$ ) under dynamic loads to establish a mathematical model to determine the ultimate load capacity estimate based on the assumption that the seismic force is quasi-static in nature and at the same time according to the dynamic method taking into account the increase by the vibrating earth mass. This artical presents an approach to calculating the design of shallow foundations subjected to dynamic loads according to quasistatic, limit equilibrium analysis and seismic dynamic methods.
\end{abstract}

\section{1. Đặt vấn đề}

Sức chịu tải cực hạn của móng nông do tải trọng ngoài gây ra do tác động của tải trọng tĩnh, một số trường hợp móng nông có thể chịu tác động của tải động dưới dạng xung kích, tải điều hòa như móng máy, tải trọng động không điều hòa như nổ hạt nhân, bom đạn và động đất,... Các tải động tác dụng lên móng theo phương đứng chủ yếu như nổ hạt nhân, kích thích bể chứa chất lỏng, móng máy; theo phương ngang phần lớn do động đất. Các tải trọng động gây ra biến dạng lớn trong nền đất gây tương tác với móng đơn, móng băng, móng bè,... và có thể bị hỏng, sụp lún, trượt gây mất ổn định. Những hư hỏng nguyên nhân thông thường là do hóa lỏng, một điều kiện mà ứng suất hữu hiệu trong đất bão hòa giảm xuống gần bằng 0 . Tuy nhiên, một số hư hỏng, biến dạng, lún sụp đã xảy ra theo quan sát từ các điều kiện hiện trường cũng cho thấy rằng chỉ có trường hợp đất bão hòa một phần, do đó có thể nói hóa lỏng là một hiện tượng cũng khó giải thích. Những năm gần đây các công trình thiết kế được quan tâm hơn về tác động và nguyên nhân gây ra do động đất. Động đất xảy ra gây sức chịu tải của móng nông có thể giảm và tăng độ lún, độ nghiêng vấn đề đặt ra cần tính toán thiết kế kết cấu móng nông sao cho phải an toàn cho cả tải trọng tĩnh và tải trọng động do động đất từ các yếu tố ảnh hưởng đến móng nông như cường độ của đất nền suy giảm theo chu kỳ, lực quán tính ngang lớn do động đất gây cho nền bị trượt hoặc lật, đất hóa lỏng bên dưới và xung quanh nền móng có thể dẫn đến độ lún lớn và nghiêng móng hoặc nền đất bị mềm do sự phân phối lại áp lực nước lỗ rỗng gây ảnh hưởng đến mất ổn định của nền. Điển hình sức chịu tải của móng nông bị phá hỏng đã xảy ra ở thành phố Adapazari do trận động đất Kocaeli năm 1999 (Karaca (2001) [7], và Yilmaz et. al (2004)) [12]. Sự suy 
giảm khả năng chịu lực của kết cấu móng ở Adapazari được thể hiện trong Hình 1. Nhằm để tiếp cận xác định đúng đắn sức chịu tải cực hạn và ổn định móng nông, cần phân tích chuyển động theo thời gian của móng, xét một số yếu tố trong đó cần quan tâm các hệ số quan trọng như: biên độ tải xung; khoảng thời gian tác dụng của xung và độ nhạy về ứng suất và biến dạng của đất trong quá trình biến dạng.

Dự đoán sức chịu tải của móng nông là một vấn đề rất quan trọng trong kỹ thuật Địa kỹ thuật, và trong những thập kỷ qua các giải pháp sử dụng phân tích giới hạn, đường trượt, cân bằng giới hạn và gần đây là phương pháp số (các phương pháp phần tử hữu hạn và phương pháp sai phân hữu hạn,v.v... đã được phát triển. Bài viết trình bày mô hình tính và cơ sở lý thuyết tính toán tiếp cận tính toán giải tích theo quan điểm tựa tĩnh và động lực học, làm cơ sở cho bài viết tiếp theo sử dụng phương pháp PTHH nghiên cứu phát triển tính toán kết cấu móng nông chịu tải trọng động trong môi trường nền đất yếu làm việc đồng thời.

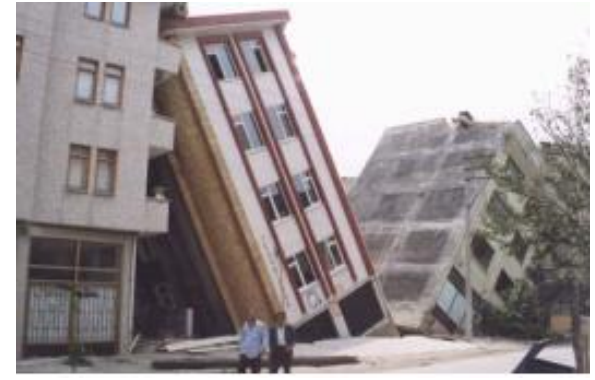

a)

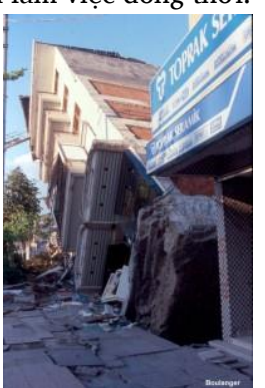

b)
Hình 1. a) Sức chịu tải bị phá hỏng của móng nông ở Adapazari; b) nền móng của tòa nhà bị lật. Tòa nhà có tỷ lệ chiều cao trên chiều rộng tương đối lớn.

\section{Phân tích phương pháp}

2.1. Ước tính sức chịu tải móng nông chịu tác động tải trọng tĩnh

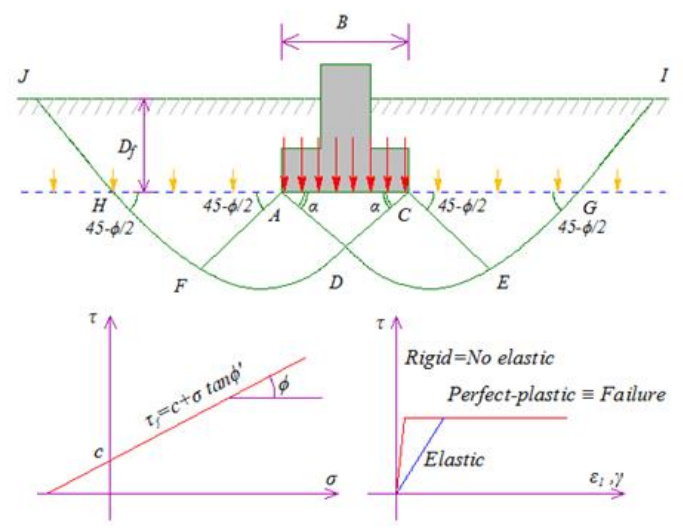

Hình 2. Mô hình mặt trượt dưới đáy móng theo Terzaghi.

Vấn đề xác định sức chịu tải theo tải trọng tĩnh của móng nông đã được nghiên cứu sâu rộng trong quá khứ bởi nhiều nhà khoa học như Terzaghi (1943), Meyerhof (1963), Hansen (1970), Vesic (1973), Kumar (2003), Dewaikar và Mohapatro (2003) và các nhà nghiên cứu khác. Sức chịu tải cực hạn của đất nền được biểu thị bằng tổ hợp tuyến tính của ba hệ số sức chịu tải $\mathrm{N}_{\mathrm{c}}, \mathrm{N}_{\mathrm{q}}$ và $\mathrm{N}_{\gamma}$ là hàm phụ thuộc duy nhất vào góc nội ma sát của đất, $f(\varphi)$. Sức chịu tải cực hạn được biểu diển ở dạng tổng quát, có xét đến các yếu tố hiệu chỉnh, hình dạng của nền móng, tải trọng và độ nghiêng của mặt đất cũng như độ sâu và độ nghiêng của bề mặt chịu lực.

$$
q_{u l t}=c \cdot N_{c} \cdot F_{c s} \cdot F_{c d} \cdot F_{c i}+q \cdot N_{q} \cdot F_{q s} \cdot F_{q d} \cdot F_{q i}+0,5 \cdot \gamma \cdot B \cdot N_{\gamma} \cdot F_{\gamma s} \cdot F_{\gamma d} \cdot F_{\gamma i}
$$

Các hệ số trong công thức (1) được thể hiện trong Bảng 1.

\section{2. Ước tính sức chịu tải cực hạn theo phương pháp tựa tĩnh}

Thiết kế nền móng trong phạm vi các khu vực dễ xảy ra động đất đòi hỏi cách tiếp cận thiết kế khác nhau người thiết kế xét đồng thời các tác động do tải trọng động đất, tĩnh tải và hoạt tải thông thường được xem xét trong phân tích tĩnh. Phương pháp thiết kế quan điểm theo tựa tĩnh là phương pháp cân bằng giới hạn hoặc phương pháp tĩnh tương đương, nghĩa là xét các tải trọng động đất giả tĩnh cùng với các lực tĩnh khác đã được sử dụng như một phương pháp đơn giản để thiết kế móng nông trong các khu vực địa chấn. Khi xảy ra động đất làm giảm khả năng chịu lực của lớp đất bên dưới và tăng độ lún và độ nghiêng là những nguyên nhân chính dẫn đến hỏng móng nông khi chịu tải trọng động đất. Vì vậy, vấn đề cần quan tâm là xác định các tham số động của môi trường đất nền, sự tương tác giữa kết cấu - môi trường nền và ứng xử động của nền đất để xác định bản chất của hư hỏng từ đó ước tính sức chịu tải địa chấn của móng càng chính xác càng tốt. Một cách tiếp cận thiết kế tốt sẽ yêu cầu xem xét tất cả các yếu tố, hệ số của các tham số của đất nền; hư hỏng, hoá lỏng do địa chấn; bản chất của tải trọng tác dụng và tương tác giữa kết cấu và đất nền địa chấn để ước tính hiệu quả sức chịu tải của móng.

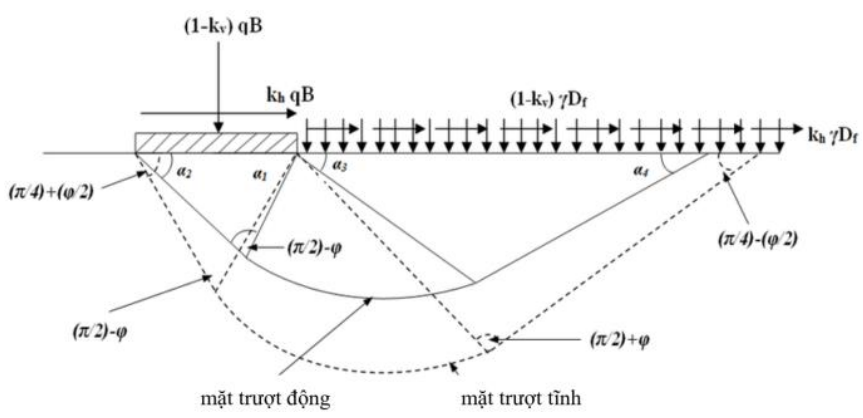

Hình 3. Mặt trượt dưới đáy móng.

Phương pháp tựa tĩnh là kỹ thuật phân tích sử dụng phương pháp cân bằng giới hạn, trên cơ sở mặt trượt của nền đất dưới đáy

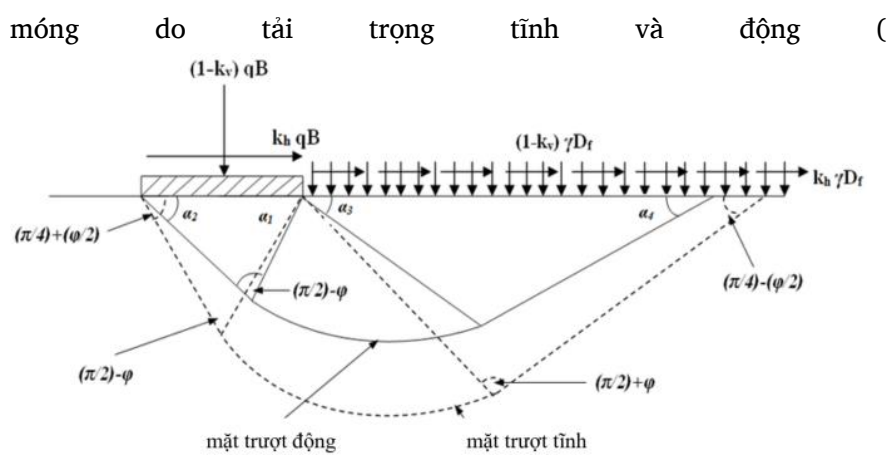


Hình 3), trong đó các lực quán tính tạo ra trên kết cấu do sự rung chuyển của mặt đất được tính đơn giản bởi một lực ngang và dọc một chiều tương đương. Các lực tương đương là khối lượng nhân với hệ số của gia tốc cho cả phương ngang và phương thẳng đứng. Các hệ số này được gọi là hệ số gia tốc, $\mathrm{K}_{\mathrm{h}, \mathrm{E}}$ và $\mathrm{K}_{\mathrm{v}, \mathrm{E}}$ tương ứng với phương ngang và phương thẳng đứng. Từ phương trình (1), sức chịu tải cực hạn của móng nông (2):

$$
q_{u l t}=c \cdot N_{c} \cdot F_{c s} \cdot F_{c d} \cdot F_{c E}+q \cdot N_{q} \cdot F_{q s} \cdot F_{q d} \cdot F_{q E}+0,5 \cdot \gamma \cdot B \cdot N_{\gamma} \cdot F_{\gamma s} \cdot F_{\gamma d} \cdot F_{\gamma E}
$$

với $\mathrm{F}_{\mathrm{cE}}, \mathrm{F}_{\mathrm{qE}}, \mathrm{F}_{\gamma \mathrm{E}}$ - các hệ số động phụ thuộc hình dạng, độ sâu và độ nghiêng được xác định.

\section{Bảng 1.}

Hệ số hình dạng, độ sâu, độ nghiêng.

Hệ số

$\begin{array}{rlrl}F_{c s} & =1+\frac{B}{L} \frac{N_{q}}{N_{c}} & & \\ \text { Hình dạng } & F_{q s} & =1+\frac{B}{L} \operatorname{tg} \varphi & \text { De Beer (1970) } \\ F_{\gamma s} & =1-0,4 \frac{B}{L} & \end{array}$

Trường hợp (a):

$D_{f} / B \leq 1$

$F_{c d}=1+0,4 D_{f} / B$

$F_{c d}=1+2 \operatorname{tg} \varphi(1-\sin \varphi)^{2} D_{f} / B$

Độ sâu

$$
F_{\gamma d}=1
$$

Trường hợp (b):

$D_{f} / B>1$

$F_{c d}=1+0,4 \operatorname{tg}^{-1}\left(D_{f} / B\right)$

$F_{c d}=1+2 \operatorname{tg} \varphi(1-\sin \varphi)^{2} \operatorname{tg}^{-1}\left(D_{f} / B\right)$

$F_{\gamma d}=1$

Độ nghiêng

$$
\begin{gathered}
F_{c i}=F_{q i}=\left(1-\frac{\beta^{o}}{90^{o}}\right)^{2} \\
F_{\gamma i}=\left(1-\frac{\beta}{\varphi}\right)^{2}
\end{gathered}
$$

với $\beta=$ là góc nghiêng của tải trọng trên móng so với đường thẳng đứng

$$
\begin{array}{r}
F_{c E}=\exp \left(-4,3 \cdot \mathrm{k}_{h, E}^{1+D}\right) \\
F_{q E}=\left(1-\mathrm{k}_{v, E}\right) \cdot \exp \left[-\left(\frac{5,3 \mathrm{k}_{h, E}^{1,2}}{1-\mathrm{k}_{v, E}}\right)\right] \\
\Rightarrow N_{q E}=N_{q} \cdot F_{q E} \\
F_{\gamma E}=\left(1-\frac{2}{3} \mathrm{k}_{v, E}\right) \exp \left[-\left(\frac{9 \mathrm{k}_{h, E}^{1,2}}{1-\mathrm{k}_{v, E}}\right)\right] \\
\Rightarrow N_{\gamma E}=N_{\gamma} \cdot F_{\gamma E}
\end{array}
$$

trong đó: $\mathrm{k}_{\mathrm{h}, \mathrm{E}}$ và $\mathrm{k}_{\mathrm{v}, \mathrm{E}}$ - hệ số gia tốc theo phương ngang và phương đứng phụ thuộc vào các tỉ số $\mathrm{N}_{\mathrm{c}, \mathrm{E}} / \mathrm{N}_{\mathrm{c}} ; \mathrm{N}_{\mathrm{q}, \mathrm{E}} / \mathrm{N}_{\mathrm{q}}$ và $\mathrm{N}_{\gamma, \mathrm{E}} / \mathrm{N}_{\gamma}$ theo góc nội ma sát $(\varphi)[1]$ và được xác định theo các đồ thị sau.

Phương trình rút gọn có dạng:

$$
q_{u l t}=c \cdot N_{c E} \cdot F_{c s} \cdot F_{c d}+q \cdot N_{q E} \cdot F_{q s} \cdot F_{q d}+0,5 \cdot \gamma \cdot B \cdot N_{\gamma E} \cdot F_{\gamma s} \cdot F_{\gamma d}
$$

Hệ số $\mathrm{k}_{\mathrm{v}, \mathrm{E}}$ được xác định theo phương trình (7)

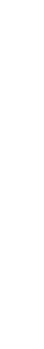

\section{.}

\section{a.} a.

. Đối với đất dính

Mô hình tính sức chịu chịu tải cực hạn của móng nông đặt trên nền đất bão hoà, điều kiện không thoát nước $\left(\varphi=0, c=c_{u}\right)$, biểu thức xác định sức chịu tải tĩnh cực hạn của móng nông theo phương đứng được xác định.

$$
q_{u l t}=c_{u} \cdot N_{c} \cdot F_{c s} \cdot F_{c d}+q \cdot N_{q} \cdot F_{q s} \cdot F_{q d}
$$


với:

$\mathrm{N}_{\mathrm{c}}, \mathrm{N}_{\mathrm{q}}$, - hệ số khả năng chịu tải là hàm của góc nội ma sát, $=\mathrm{f}(\varphi)$;

$\mathrm{F}_{\mathrm{cs}}, \mathrm{F}_{\mathrm{qs}}$ - hệ số hình dạng;

$\mathrm{F}_{\mathrm{cd}}, \mathrm{F}_{\mathrm{qd}}$ - hệ số chiều sâu.

Do đất bão hòa $\varphi=0$, theo bảng tính của Meyerhof (Bảng 2)

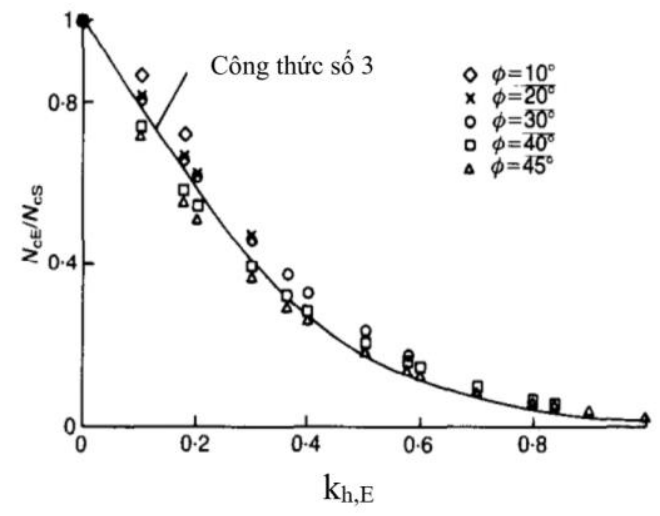

Công thức số (4) (18)

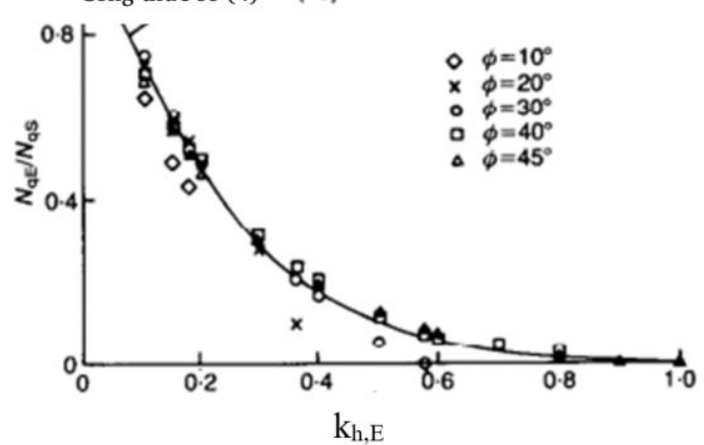

Hình 4. a) Quan hệ $\mathrm{k}_{\mathrm{h}, \mathrm{E}}$ và $\mathrm{N}_{\mathrm{cE}} / \mathrm{N}_{\mathrm{c}} ; \mathrm{k}_{\mathrm{h}, \mathrm{E}}$ và $\mathrm{N}_{\mathrm{qE}} / \mathrm{N}_{\mathrm{q}}$ theo góc nội ma sát $\varphi$ [1].

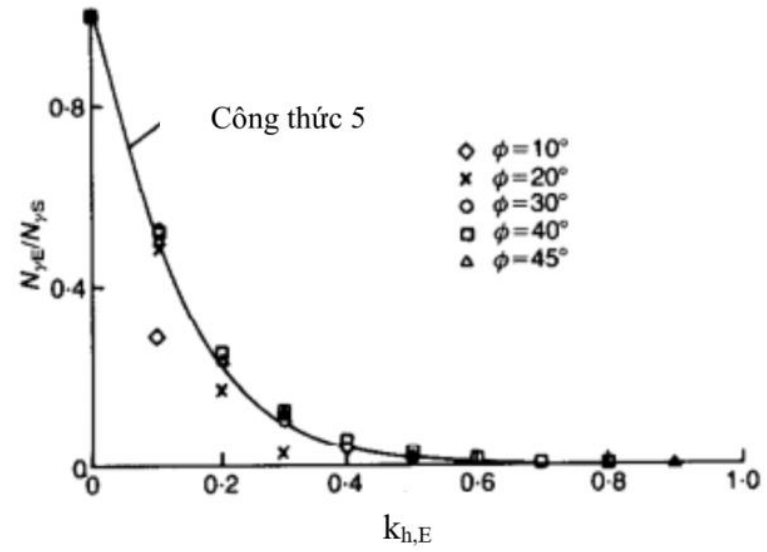

Hình 5. Quan hệ $\mathrm{k}_{\mathrm{h}, \mathrm{E}}$ và $\mathrm{N}_{\gamma \mathrm{E}} / \mathrm{N}_{\gamma}$ theo góc nội ma sát $\varphi$ [1].

Bảng 2.

Bảng tra các hệ số khả năng chịu tải theo Meyerhof.

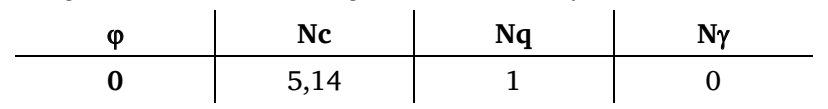

\begin{tabular}{c|c|c|c}
$\mathbf{1 0}$ & 8,35 & 2,47 & 0,37 \\
$\mathbf{1 5}$ & 10,98 & 3,94 & 1,13 \\
$\mathbf{2 0}$ & 14,83 & 6,4 & 2,87
\end{tabular}

trong đó:

$$
\begin{aligned}
& F_{c s}=1+\left(\frac{B}{L}\right)\left(\frac{N_{q}}{N_{c}}\right) \\
& \text { Khi } \varphi=0 \Rightarrow F_{c s}=1+\left(\frac{B}{L}\right)\left(\frac{N_{q}}{N_{c}}\right)=1+0,1946\left(\frac{B}{L}\right) \\
& \text { và } \mathrm{F}_{\mathrm{qs}}=1+\operatorname{tg} \varphi=1 \\
& F_{c d}=1+0,4\left(\frac{D_{f}}{B}\right) \quad \operatorname{khi}\left(\frac{D_{f}}{B}\right) \leq 1 \\
& F_{c d}=1+0,4 \cot g\left(\frac{D_{f}}{B}\right) \quad \operatorname{khi}\left(\frac{D_{f}}{B}\right)>1 \\
& \text { và } \mathrm{F}_{\mathrm{qd}}=1
\end{aligned}
$$

Từ công thức (8) ta xác định được sức chịu tải cực hạn.

$$
\begin{aligned}
& \text { Khi }\left(\frac{D_{f}}{B}\right) \leq 1: q_{u l t}=5,14 \cdot c_{u}\left[1+0,1946\left(\frac{B}{L}\right)\right]\left[1+0,4\left(\frac{D_{f}}{B}\right)\right]+q \\
& \operatorname{Khi}\left(\frac{D_{f}}{B}\right)>1: q_{u l t}=5,14 \cdot c_{u}\left[1+0,1946\left(\frac{B}{L}\right)\right]\left[1+0,4 \cdot \operatorname{cotg}\left(\frac{D_{f}}{B}\right)\right]+q
\end{aligned}
$$

Theo Carroll thực nghiệm theo sự gia tăng biến dạng đối với đất sét bão hòa không thoát nước xác định được mối quan hệ giữa $\mathrm{c}_{\mathrm{u} \text { (động) }}$ và $\mathrm{c}_{\mathrm{u} \text { (tinh) }}$ tương đương xấp xỉ 1,5 [5].

Ta xác định được lực dính không thoát nước $\mathrm{c}_{\mathrm{u}}$ (điều kiện $\varphi=$ 0) của đất nhất định tại một tốc độ biến dạng nhất định, thì có thể tính toán sức chịu tải cực hạn.

b. Đối với đất rời

Mô hình tính kết cấu móng đặt trên nền đất rời lúc này $\mathrm{c}=0$, sức chịu tải cực hạn theo tĩnh tải.

$$
q_{u l t}=q \cdot N_{q} \cdot F_{q s} \cdot F_{q d}+0,5 \cdot B \cdot N_{\gamma} \cdot F_{\gamma s} \cdot F_{\gamma d}
$$

trong đó các hệ số $\mathrm{N}_{q}, \mathrm{~N}_{\gamma}$ được xác định theo Vesic (1973) [10], như sau:

$$
N_{q}=e^{\pi \operatorname{tg} \varphi} \operatorname{tg}^{2}\left(\frac{\pi}{4}+\frac{\varphi}{2}\right) \quad \text { và } \quad N_{\gamma}=2\left(N_{q}+1\right) \operatorname{tg} \varphi
$$

Các hệ số hình dáng

$$
F_{q s}=1+\left(\frac{B}{L}\right) \operatorname{tg} \varphi
$$

$$
F_{\gamma s}=1-0,4 \cdot\left(\frac{B}{L}\right)
$$

và hệ số độ sâu 


$$
\begin{gathered}
\left\{\begin{array}{cc}
F_{q d}=1-2 \cdot \operatorname{tg} \varphi \cdot(1-\sin \varphi)^{2} \cdot\left(\frac{D_{f}}{B}\right) ; & \operatorname{khi}\left(\frac{D_{f}}{B}\right) \leq 1 \\
F_{\gamma d}=1
\end{array}\right. \\
\left\{\begin{array}{cc}
F_{q d}=1+2 \cdot \operatorname{tg} \varphi \cdot(1-\sin \varphi)^{2} \cdot \cot g\left(\frac{D_{f}}{B}\right) ; & \operatorname{khi}\left(\frac{D_{f}}{B}\right)>1 \\
F_{\gamma d}=1 &
\end{array}\right.
\end{gathered}
$$

Khi tải trọng động tác động nhanh lên nền gây ra phá hủy, khả năng chịu lực cuối cùng sẽ thay đổi. Bằng thực nghiệm Vesic, Banks và Woodward đã tiến hành một số thử nghiệm mô hình trong phòng thí nghiệm chứng minh được mối tương quan giữa tải vận tốc với hệ số sức chịu tải $\mathrm{q}_{\mathrm{u}} / 2 \gamma \mathrm{B}$, Hình 6 [5].

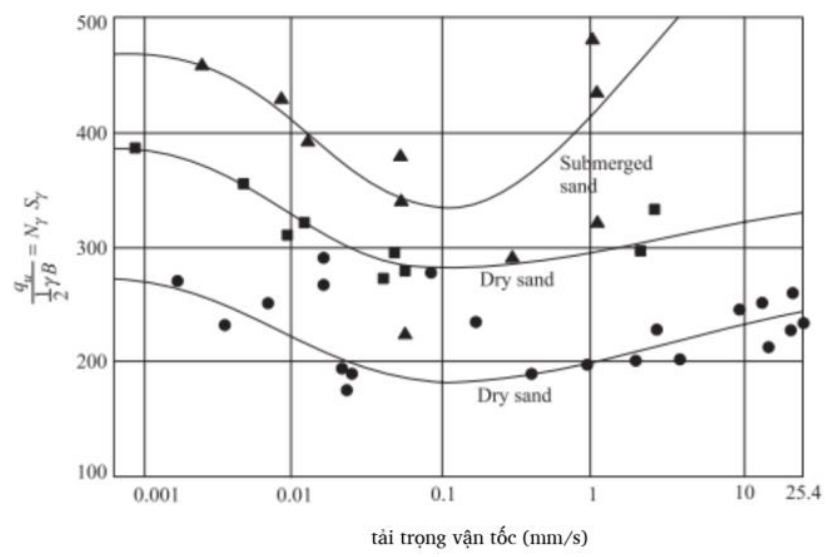

Hình 6. Mối tương quan hệ số sức chịu tải - tải trọng vận tốc [5].

Từ Hình 6 cho thấy hệ số sức chịu tải giảm đến min rồi tăng dần lên tương ứng với góc nội ma sát giảm tương ứng $2^{0}$ phù hợp tốc độ biến dạng nền do Whitman và Healy, và Shao Shengjun, Xie Dingyi nghiên cứu $[9,11]$. Tính toán sức chịu tải cực hạn của móng nông trên nền cát chịu tải trọng động với góc nội ma sát theo đề nghị của Vesic (1973) [5], theo phương trình (19), tuy nhiên chưa chính xác trong trường hợp nền cát chịu tải trọng động đất bị hóa lỏng .

$$
\varphi_{\text {động }}=\varphi-2^{0}
$$

\subsection{Phương pháp cân bằng giới hạn}

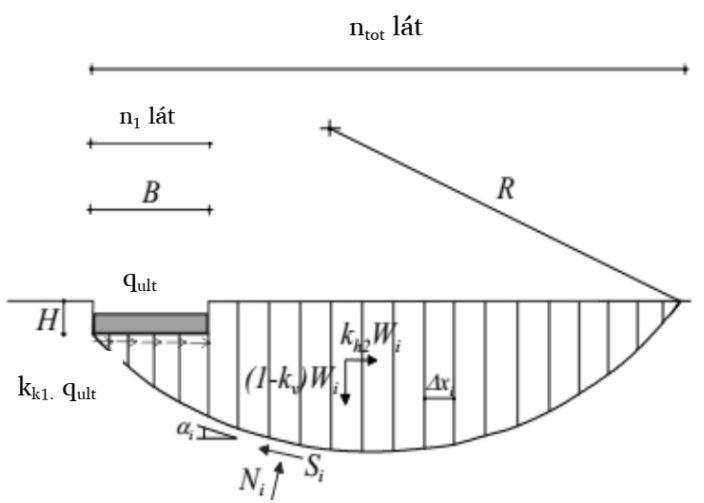

Hình 7. Chia lát mặt trượt dưới đáy móng.
Phương pháp cân bằng giới hạn dựa trên cơ chế mặt trượt (Hình 7), mô hình phân tích khả năng chịu lực của móng nông có độ sâu hạn chế. Lực tác động của động đất quan điểm là lực giả tĩnh tác dụng lên chân móng và lớp đất bên dưới móng. Sức chịu tải cực hạn có thể xác định tại một thời điểm cân bằng, tương ứng cung trượt thời điểm xét ta xác định tâm và cung trượt. Phương trình trạng thái cân bằng mô men và mô men động lực có dạng công thức (20) [2].

$$
\mathrm{M}_{\text {động }}=\sum_{i=1}^{n_{\text {tot }}} \mathrm{W}_{i}\left(1-\mathrm{k}_{v}\right) \mathrm{b}_{\mathrm{w} i}+q_{u l t} \sum_{i=1}^{n_{1}} \Delta x_{i} \cdot \mathrm{b}_{q l i}+q_{u l t} \sum_{i=1}^{n_{1}} \mathrm{k}_{h 1} \cdot \Delta x_{i} \cdot \mathrm{b}_{q l h i}+\sum_{i=1}^{n_{1}} \mathrm{k}_{h 2} \cdot \mathrm{W}_{i} \cdot \mathrm{b}_{\mathrm{w} h i}
$$

trong đó:

$\Delta x_{i}$ - bề rộng lát thứ i;

$\mathrm{W}_{\mathrm{i}}$ - trọng lượng đất lát thứ i;

R - bán kính đường tròn mặt trượt;

$\mathrm{n}_{\text {tot }}$ - tổng số lát;

$\mathrm{n}_{1}$ - số lát dưới đáy móng;

$\mathrm{k}_{\mathrm{v}}$ - hệ số động theo phương đứng cho khối lượng đất;

$\mathrm{k}_{\mathrm{h} 1}$ - hệ số động theo phương ngang cho tải giới hạn;

$\mathrm{k}_{\mathrm{h} 2}$ - hệ số động theo phương ngang cho khối lượng đất;

$\mathrm{b}_{\mathrm{wi}}$ - khoảng cách trọng lượng $\mathrm{W}_{\mathrm{i}}$ của lát thứ $\mathrm{i}$ đến tâm của đường tròn mặt trượt;

$\mathrm{b}_{\mathrm{qli}}-$ khoảng cách của tải trọng giới hạn $\mathrm{q}_{\mathrm{ult}}$ tác dụng lên lát thứ i đến tâm của đường tròn mặt trượt;

$\mathrm{b}_{\text {qhli }}$ - khoảng cách của giới hạn cắt $\mathrm{k}_{\mathrm{h} 1} \cdot \mathrm{q}_{\mathrm{ult}}$ tác dụng lên lát thứ $\mathrm{i}$ đến tâm tâm của đường tròn mặt trượt;

$\mathrm{b}_{\text {whi }}$ - khoảng cách của lực quán tính $\mathrm{k}_{\mathrm{h} 2} \cdot \mathrm{W}_{\mathrm{i}}$ tác dụng lên lát thứ i đến tâm của đường tròn mặt trượt;

Mômen kháng trượt do cường độ cắt Si tác dụng dọc theo đáy của các lát cắt được xác định.

$$
M_{\text {kháng trượt }}=R \sum_{i=1}^{n_{\text {tot }}} S_{i}=R\left(\sum_{i=1}^{n_{\text {tot }}} \frac{c \cdot \Delta x_{i}}{\cos \alpha_{i}}+\sum_{i=1}^{n_{\text {tot }}} N_{i} \cdot \operatorname{tg} \varphi_{i}\right)
$$

với: $\alpha_{i}-$ góc tại đáy của lát thứ $i$;

$\mathrm{N}_{\mathrm{i}}$ - sự phân bố ứng suất tác động lên đáy của lát cắt thứ $\mathrm{i}$, theo Bishop (1955) phương trình cân bằng theo phương đứng ta có:

$$
\begin{aligned}
& N_{i}=\frac{q_{u l t} \cdot \Delta x_{i}+\mathrm{W}_{i}\left(1-\mathrm{k}_{v}\right)-\mathrm{c} \cdot \Delta x_{i} \operatorname{tg} \alpha_{i}}{\cos \alpha_{i}+\sin \alpha_{i} \cdot \operatorname{tg} \varphi_{i}} \quad \text { khi } 1 \leq n<n_{1} \\
& N_{i}=\frac{\mathrm{W}_{i}\left(1-\mathrm{k}_{v}\right)-\mathrm{c} \cdot \Delta x_{i} \operatorname{tg} \alpha_{i}}{\cos \alpha_{i}+\sin \alpha_{i} \cdot \operatorname{tg} \varphi_{i}} \quad \text { khi } \quad n_{1}+1 \leq i \leq n_{t o t}
\end{aligned}
$$


Điều kiện móng ổn định khi chịu tác động của tải trọng động, từ phương trình (20) và (21) để ta có phương trình cân bằng:

$$
\begin{aligned}
& \mathrm{M}_{\text {động }}=\mathrm{M}_{\text {kháng trượt }}
\end{aligned}
$$

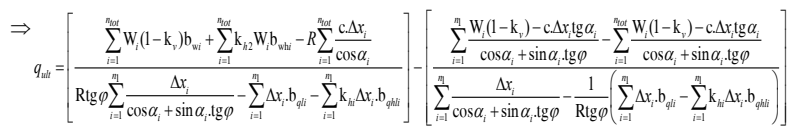

\subsection{Phân tích động lực học}
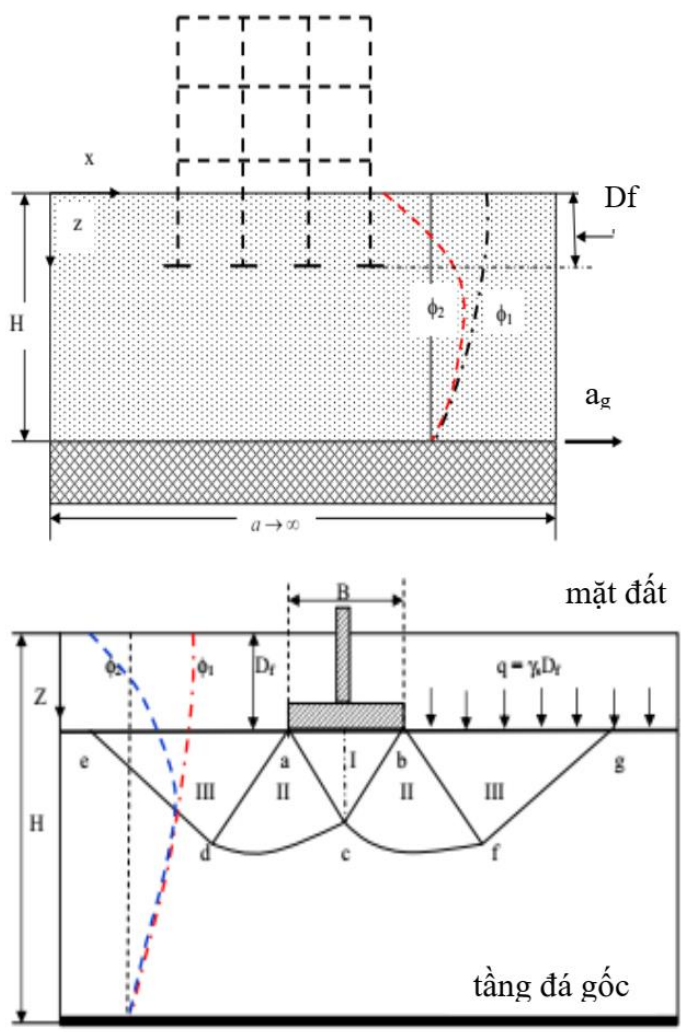

Đáy móng

a) Kết cấu chịu tải trọng động đất

b) Móng nông chịu tải trọng động đất

Hình 8. Móng chịu tải trọng động với gia tốc tại nền đá cứng.

Khi xả ra động đất gây biến dạng nền đất thông qua "trường tự do" (free field) tương ứng chu kì $\mathrm{T}$ khi đó ta xác định được các hàm dáng.

$$
\phi_{n}=\cos (2 n-1) \frac{\pi z}{2 \mathrm{H}}
$$

Ta xác định dịch chuyển khi xảy ra dao động tại độ sâu z.

$$
u_{n z}=-k_{n} \cdot C_{f} \cdot \cos (2 n-1) \frac{\pi z}{2 \mathrm{H}}
$$

trong đó: $k_{n}$ - hệ số tham gia rung động đất, [4];

$C_{f}$ - nhân tố mã số; phụ thuộc vào vùng tính và hệ số ứng xử của đất nền [3];

Và gia tốc nền tại đáy móng $\left(\mathrm{z}=D_{f}\right)$.

$$
\ddot{u}_{n z}=k_{n} \cdot C_{f} \cdot \cos (2 n-1) \frac{\pi D_{f}}{2 \mathrm{H}}
$$

Hệ số lực động theo phương ngang và phương đứng như sau.

$$
\begin{array}{r}
\alpha_{n, H}=k_{n} \cdot C_{f} \cdot \cos (2 n-1) \frac{\pi D_{f}}{2 \mathrm{H}}\left(\frac{S_{a n}}{g}\right) \\
\alpha_{n, V}=\frac{2}{3} \alpha_{n, H}
\end{array}
$$

Dưới tải trọng ngoài bên trên tạo ra mặt trượt hỏng của của đất theo cung edcfg được hiển thị Hình 8 và sức chịu tải cực hạn khi chịu tải trọng động có dạng.

$$
\begin{aligned}
q_{u t t}=c & {\left[N_{c} \pm \alpha_{n, H}\left(\frac{\gamma B}{c}\right) \cdot f(\alpha)\right]+q\left[N_{q} \pm \alpha_{n, H}\left(\frac{\gamma B}{q}\right) \cdot f(\alpha)\right]+\frac{1}{2} \gamma B\left[N_{\gamma} \pm \alpha_{n, H}\left(\frac{\gamma B}{\frac{1}{2} \gamma B}\right) \cdot f(\alpha)\right] } \\
& \text { với } f(\alpha)=\left(5 \operatorname{tg} \alpha+2 \operatorname{tg}^{2} \alpha\right) / 6
\end{aligned}
$$

Tải trọng cho phép của móng khi chịu tải trọng động đất.

$$
q_{\text {allow }}=\frac{\left[c N_{c}+q N_{q}+\frac{1}{2} \gamma B N_{\gamma}\right]}{F S} \pm 3 \alpha_{n, H} \gamma B f(\alpha)=\frac{q_{\text {allow, tinh }}}{F S} \pm 3 \alpha_{n, H} \gamma B f(\alpha) \text { (31) }
$$

hay:

$q_{\text {allow }}=\left(\frac{\mathrm{N}}{\mathrm{F}} \pm \frac{\mathrm{M}}{\mathrm{Z}_{f}}\right) \pm 3 k_{n}\left(\frac{\mathrm{ZI}}{2 \mathrm{R}}\right) \cos \left[(2 n-1) \frac{\pi D_{f}}{2 \mathrm{H}}\right]\left(\frac{S_{a n}}{g}\right) \gamma B\left(\frac{5 \mathrm{tg}\left(45+\frac{\varphi}{2}\right)+2 \operatorname{tg}^{2}\left(45+\frac{\varphi}{2}\right)}{6}\right)$

trong đó:

$$
\begin{aligned}
& \mathrm{N} \text { - tải trọng từ bên trên kêt cấu truyền xuống móng; } \\
& \mathrm{F} \text { - diện tích móng; } \\
& \mathrm{M} \text { - mô men tại móng từ bên trên truyền xuống; } \\
& \mathrm{Z}_{\mathrm{f}} \text { - mô đun móng. }
\end{aligned}
$$

\section{Kết luận}

Sự phức tạp đất nền khi chịu tác động của động đất gây làm giảm sức chịu tải của móng nông và tăng độ lún, nghiêng của kết cấu móng. Phần lớn tập trung các thành phần đáng kể như: lực quán tính gây ra bởi lực tác dung ngang ở kết cấu công trình và tải trọng động làm chuyển vị và biến dạng của đất nền tương tác lên kết cấu móng.

Trên cơ sở phương pháp đánh giá khả năng chịu lực của đất đã được thiết lập bởi Terzaghi, Meyerhof, v.v., Bài báo tiếp tục xác định sức chịu tải cực hạn của móng khi chịu tải trọng động tương ứng dưới dạng đất nền mềm chưa hóa lỏng và hóa lỏng trên nền đá cứng. Khi tính toán cần được đánh giá cẩn thận và khuyến nghị khả năng chịu tải của nền theo tải trọng cho phép và cực hạn.

Đối với một số vùng thiết kế thiếu gia tốc đỉnh của nền ta có thể sử dụng phương pháp tựa tĩnh và phương pháp cân bằng giới hạn cho bước thiết kế cơ sở. Trường hợp có gia tốc đỉnh của nền ta có thể sử dụng phương pháp động lực học tính toán hoặc sử dụng phương pháp 
số giải bài toán cho lời giải tương tác kết cấu móng với môi trường đất mềm sát với bài toán thực tiễn hơn.

\section{Tài liệu tham khảo}

[1]. Budhu, M., and Al-Karni, A. (1993). Seismic bearing capacity of soils. Géotechnique, 43(1), 181-187.

[2]. Castelli, F. \& Motta, E. (2003). Bearing capacity of shallow foundations resting on a soil layer of limited depth. Proceedings International Symposium on Shallow Foundations. Paris: FONDSUP2003.

[3]. Chowdhury I, Dasgupta SP (2007). Dynamic earth pressure on rigid unyielding walls under earthquake forces. India: Indian Geotech J37(2):81-93.

[4]. Chowdhury I, Ghosh A, Dasgupta SP (2015). Stiffness degradation and damping augmentation of soil under earthquake loading. Oklahoma, USA: Electron J Geotech Eng.

[5]. Das B. M., and Ramana G. V. (2011). Principle of soil Dynamic. second edition. Cengage Learning 200.

[6]. Eurocode 8: Design of structures for earthquake resistance Part 5: Foundations, retaining structures and geotechnical aspects.

[7]. Karaca, G. (2001), An Investigation into Large Vertical Displacement Experienced by the Structures in Adapazari during 17 August 1999 Earthquake. Ankara, Turkey: MS thesis, Middle East Technical University.

[8]. Richards, R., Jr., Elms, D. G., and Budhu, M. (1993). Seismic Bearing Capacity of and Settlements of Foundations. Journal of Geotechnical Engineering Division, ASCE, Vol. 119, No. 4, pp. 662-674.

[9]. Shao Shengjun, Xie Dingyi (2002), Dynamic effective shear strength of saturated sand. ACTA MECHANICA SINICA (English Series), Vo1.18, No.4

[10]. Shenkman, S., and Mckee, K. E. (1961). Bearing Capacity of Dynamically Loaded Footings. USA: Special Technical Publication No. 305, American Society for Testing and Materials, pp. 78-90.

[11]. Whitman, R. V., and Healy, K. A. (1962). Shear Strength of Sands During Rapid Loadings, Journal of the Soil Mechanics and Foundations Division, ASCE, Vol. 88, No. SM2, pp. 99-132.

[12]. Yilmaz M. Tolga and Bakir B. Sadik.(2009). Capacity of Shallow Foundations on Saturated Cohesionless Soils under Combined Loading. Can. Geotech. J. Vol. 46. 